\title{
Importante éclosion communautaire d'oreillons au Manitoba (Canada) entre septembre 2016 et décembre 2018
}

\author{
Yichun Wei ${ }^{1 *}$, Krista Wilkinson², Richard Rusk², Kamran Kadkhoda ${ }^{4,5}$, Carla Loeppky1,6
}

\section{Résumé}

Contexte : Après la mise en œuvre des programmes d'immunisation systématique contre les oreillons au Manitoba à la fin des années 1980, le taux d'incidence de la maladie était faible, avec zéro à neuf cas enregistrés chaque année. En septembre 2016, une éclosion d'oreillons s'est déclarée chez des étudiants universitaires pleinement vaccinés à Winnipeg, au Manitoba.

Objectifs : Nous décrivons ici l'enquête sur cette éclosion d'oreillons survenue à l'échelle provinciale entre septembre 2016 et décembre 2018. Nous présentons également de manière détaillée les mesures de santé publique mises en œuvre et les difficultés rencontrées. Nous précisons enfin les facteurs ayant pu contribuer à la transmission soutenue du virus.

Méthodes : Les cas d'oreillons probables et confirmés ont été étudiés par les services de santé publique à l'aide du formulaire d'enquête élaboré dans le cadre de cette éclosion. Les cas d'oreillons confirmés ont été liés au registre provincial d'immunisation. Une équipe d'intervention contre l'éclosion a planifié et mis en œuvre des mesures de contrôle sur l'ensemble de la province.

Résultats : L'éclosion s'est déclarée chez des étudiants universitaires vaccinés en septembre 2016 puis s'est propagée à toute la province. L'activité était élevée et soutenue dans les régions éloignées du Nord. Fin 2018, 2223 cas avaient été confirmés. Tous les groupes d'âge étaient touchés, et l'incidence était la plus élevée chez les personnes de 18 à 29 ans. Le taux de couverture des cas confirmés avec l'administration de deux doses du vaccin contenant le virus des oreillons était proche de $70 \%$.

Conclusion : Cette éclosion prolongée a mis au jour une vaste population dont la vulnérabilité résultait probablement d'une sous-vaccination et d'une baisse de l'immunité conférée par la vaccination en l'absence d'une stimulation naturelle résultant d'une exposition au virus des oreillons. Il est important de maintenir un taux de couverture vaccinale élevé avec l'administration de deux doses du vaccin contenant le virus des oreillons. La possibilité $d^{\prime}$ administrer une troisième dose du vaccin contenant le virus des oreillons pourrait être envisagée lors de futures éclosions.
Cette oeuvre est mise à la disposition selon les termes de la licence internationale Creative Commons Attribution 4.0

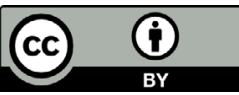

Affiliations

1 Épidémiologie et surveillance, Santé, Aînés et Vie active Manitoba, Winnipeg, MB

${ }^{2}$ Santé de la population et du public, Office régional de la santé de Winnipeg, Winnipeg, MB

${ }^{3}$ Lutte contre les maladies transmissibles, Santé, Aînés et Vie active Manitoba, Winnipeg, MB

${ }^{4}$ Laboratoire provincial de Cadham, Winnipeg, MB

${ }^{5}$ Immunopathology Laboratory, Cleveland Clinic-Main Campus, Cleveland, $\mathrm{OH}$

${ }^{6}$ Department of Community Health Sciences, University of Manitoba, Winnipeg, MB

\section{*Correspondance :} joy.wei@gov.mb.ca

Citation proposée : Wei Y, Wilkinson K, Rusk R, Kadkhoda K, Loeppky C. Importante éclosion communautaire d'oreillons au Manitoba (Canada) entre septembre 2016 et décembre 2018. Relevé des maladies transmissibles au Canada 2020;46(4):80-6. https://doi.org/10.14745/ccdr.v46i04a03f

Mots-clés : oreillons, éclosion, incidence, vaccination, vaccin ROR, enquête, immunité, Manitoba

\section{Introduction}

Les oreillons sont une maladie causée par le virus des oreillons, qui appartient à la famille des Paramyxoviridae. Les symptômes d'une infection aux oreillons comprennent notamment la fièvre, les maux de tête, ainsi qu'une tuméfaction et une sensibilité caractéristiques de la glande parotide ou d'une autre glande salivaire. La méningite aseptique, l'encéphalite,
I'orchite, l'oophorite, la surdité et la pancréatite sont de rares complications imputables à l'infection aux oreillons (1-3). Au Manitoba, les cas confirmés en laboratoire et les cas probables doivent être déclarés à Santé, Aînés et Vie active Manitoba (SAVAM), conformément à la Loi sur la santé publique du Manitoba (4). 
L'administration d'une dose unique du vaccin contre la rougeole, les oreillons et la rubéole (ROR) aux enfants âgés de douze mois a été ajoutée au calendrier de vaccination systématique des enfants au Manitoba en 1983. L'administration d'une seconde dose du vaccin ROR aux enfants âgés de quatre à six ans a été ajoutée au calendrier de vaccination systématique en 1996 (5). Ces pratiques sont conformes à la dernière recommandation formulée par le Comité consultatif national de l'immunisation (CCNI) en 2007, au sujet du vaccin contenant le virus des oreillons. Après avoir étudié les éclosions d'oreillons survenues au Canada et dans le reste du monde, le CCNI a recommandé l'administration systématique de deux doses du vaccin contre les oreillons chez les nourrissons et les enfants, ainsi que chez certains groupes d'adultes à risque élevé, notamment les étudiants du secondaire et du postsecondaire, le personnel militaire et les travailleurs de la santé (6).

L'incidence des oreillons a continuellement été faible au Manitoba, avec zéro à neuf cas recensés chaque année entre 2000 et 2015 , soit, en moyenne, 0,3 cas pour 100000 personnes (7). La maladie touche surtout la tranche des 18 à 45 ans. Le taux d'incidence moyen des oreillons au Canada était de 0,3 cas pour 100000 personnes entre 2011 et 2015 (8).

Le 18 octobre 2016, les services de surveillance de routine ont détecté une série de six cas d'oreillons dont les symptômes sont apparus entre le 25 septembre et le 12 octobre 2016, à Winnipeg, la capitale du Manitoba. II s'agissait de six étudiants de l'Université du Manitoba âgés de 18 à 24 ans. Trois d'entre eux faisaient partie des équipes sportives de l'université. Tous ces étudiants avaient reçu deux doses du vaccin ROR dans leur enfance, preuves à l'appui. SAVAM a déclaré une éclosion d'oreillons et constitué une équipe d'intervention contre l'éclosion dans la même journée. Cinq bureaux régionaux sanitaires fournissent des services de santé dans les cinq secteurs géographiques financés par les fonds publics au Manitoba : une région urbaine, l'Office régional de la santé de Winnipeg, et quatre régions rurales, l'Office régional de la santé d'Entre-les-Lacs et de l'Est, Southern Health-Santé Sud, Santé de Prairie Mountain et l'Office régional de la santé du Nord (9). Tous les offices régionaux de la santé ont rejoint l'équipe d'intervention.

Nous décrivons dans ce rapport l'enquête sur cette éclosion d'oreillons survenue à l'échelle provinciale entre septembre 2016 et décembre 2018. Nous présentons également de manière détaillée les mesures de santé publique mises en œuvre et les difficultés rencontrées. Nous précisons enfin les facteurs ayant pu contribuer à la transmission soutenue du virus.

\section{Méthodes}

Une éclosion provinciale d'oreillons a été déclarée le 18 octobre 2016, et sa fin a été confirmée le 31 décembre 2018, lorsque le niveau d'activité est revenu à la normale. Cette éclosion s'est déclarée chez une population universitaire, mais s'est rapidement répandue jusqu'à atteindre un niveau soutenu à l'échelle de toute la province. L'équipe d'intervention contre l'éclosion a planifié et mis en œuvre des mesures de contrôle, notamment la recherche rapide des contacts, la recommandation d'autoisolement après l'apparition des symptômes, la communication (dont la diffusion d'une documentation pédagogique) et la mise à disposition du vaccin ROR aux contacts réceptifs.

\section{Enquête épidémiologique}

Les définitions de cas étaient tirées du Protocole provincial de gestion des maladies transmissibles applicable aux oreillons (10). Un cas probable était caractérisé par l'apparition de symptômes compatibles avec les oreillons (apparition brutale d'une tuméfaction unilatérale ou bilatérale, douloureuse, spontanément résolutive de la glande parotide ou d'une autre glande salivaire) pendant deux jours ou plus, et ce, le ou après le $1^{\text {er }}$ septembre 2016 . Un cas confirmé était caractérisé par une confirmation en laboratoire d'une infection récente aux oreillons dans un cas probable ou un cas probable ayant un lien épidémiologique avec un cas confirmé en laboratoire, et en l'absence de l'administration récente du vaccin contenant le virus des oreillons. Le Protocole de gestion des maladies transmissibles de SAVAM recommande aux enquêteurs de consulter la version actuelle du Guide canadien d'immunisation pour se renseigner sur les réactions et les délais propres à chaque tranche d'âge et ainsi déterminer si les symptômes pouvaient être imputables à l'administration récente du vaccin contenant le virus des oreillons.

Un formulaire d'enquête sur l'éclosion a été élaboré pour recueillir des renseignements sur les caractéristiques démographiques, la profession, les symptômes, les complications et leur gravité, la situation vaccinale et les activités effectuées durant la période d'incubation (douze à vingt-cinq jours avant l'apparition de la parotidite) et la période de transmissibilité (sept jours avant et jusqu'à cinq jours après l'apparition de la parotidite). Les offices régionaux de la santé ont immédiatement lancé une enquête sur l'éclosion et mis en œuvre des mesures de contrôle. Des infirmières régionales de la santé publique ont interrogé les sujets atteints et rempli les formulaires d'enquête avant de les soumettre à SAVAM.

SAVAM a coordonné les activités de collecte et de saisie des données. Des renseignements généraux sur l'ensemble des signalements de cas d'oreillons ont été saisis dans la base de données de surveillance de routine. Une base de données réservée aux éclosions a été créée pour y consigner les renseignements issus des formulaires d'enquête sur l'éclosion. En 
raison du nombre élevé de signalements de cas d'oreillons, seuls les cas confirmés ont été saisis dans la base de données. Les cas confirmés ont été liés au registre provincial d'immunisation afin de calculer le taux de couverture vaccinale. Nous avons entrepris une analyse descriptive pour déterminer les caractéristiques épidémiologiques et géographiques de l'éclosion. La mise en relation et l'analyse des données ont été effectuées à l'aide de SAS Enterprise Guide, version 7.1 (SAS Institute Inc., Gary, Californie du Nord, États-Unis).

\section{Analyses en laboratoire}

Le Laboratoire provincial de Cadham à Winnipeg a réalisé des analyses cliniques standards sur des échantillons, notamment des analyses visant à dépister les anticorps de l'immunoglobine $M(\operatorname{lgM})$ et de l'immunoglobine $\mathrm{G}(\operatorname{lgB})$ contre le virus des oreillons dans les échantillons de la phase aiguë et de la phase de convalescence à environ sept à dix jours d'écart; des analyses visant à détecter l'acide ribonucléique du virus des oreillons au moyen de la technique de transcription inverse suivie de réaction en chaîne de la polymérase (RT-PCR); et des essais visant à isoler le virus des oreillons dans un milieu de culture. Les échantillons de cultures positives ont été envoyés au Laboratoire national de microbiologie (LNM) aux fins de génotypage viral.

\section{Couverture vaccinale}

Les données de vaccination ont été extraites du registre provincial d'immunisation de la population à partir du Système de gestion de l'information sur la santé publique (SGISP). Ce registre a été mis en place en 1988 pour consigner les vaccinations des personnes nées au Manitoba à partir du 1 er janvier 1980. Les vaccinations effectuées en dehors du Manitoba ne sont pas consignées sans document officiel à l'appui. De ce fait, les dossiers de vaccination sont généralement plus complets chez les personnes de moins de trente ans ou nées après 1986 qui ont grandi au Manitoba.

\section{Interventions}

Les mesures de contrôle comprenaient notamment la recherche rapide des contacts, les infirmières régionales de la santé publique étant chargées d'assurer la gestion des cas et des contacts. L'auto-isolement pendant cinq jours suivant l'apparition des symptômes était recommandé pour les cas symptomatiques. Les contacts susceptibles d'avoir été exposés durant la période de transmissibilité ont été avertis. Ils ont également été sensibilisés sur les oreillons, notamment sur les premiers signes et symptômes de la maladie, et ont été invités à consulter un professionnel de la santé dans le cas où ces symptômes se manifesteraient.

Le vaccin ROR a été proposé aux contacts réceptifs (personnes nées entre 1970 et 1984 auxquelles aucune dose de vaccin contenant le virus des oreillons n'a été officiellement administrée et personnes nées après 1984 auxquelles moins de deux doses ont été officiellement administrées) conformément aux critères d'admissibilité (11) du Manitoba et à la dernière recommandation du CCNI (6). Les établissements de santé étaient encouragés à s'assurer que tous leurs effectifs étaient vaccinés. Dans les établissements correctionnels touchés par des cas d'oreillons, des séances de vaccination ont été organisées à l'intention du personnel et des détenus réceptifs.

SAVAM a diffusé une documentation pédagogique auprès des universités, des écoles et du grand public. Une série de courriers ont été envoyés aux universités, aux écoles, aux garderies et aux organismes sportifs du Manitoba pour sensibiliser davantage le public. SAVAM a également répondu aux demandes de renseignements des médias et publié des communiqués pour faire le point sur l'éclosion et souligner l'importance de la vaccination. Des mises à jour hebdomadaires ont été publiées sur un site Web public géré par SAVAM. Des courriers ont été envoyés aux fournisseurs de soins de santé pour orienter les pratiques de prévention et de contrôle. SAVAM a diffusé des alertes de santé publique par l'intermédiaire du Réseau canadien de renseignements sur la santé publique (RCRSP) pour avertir ses homologues provinciaux et fédéraux du secteur de la santé publique.

En janvier 2018, l'équipe d'intervention contre l'éclosion a envisagé la possibilité de proposer une troisième dose du vaccin $R O R$ dans la région de l'Office régional de la santé du Nord, après que le Comité consultatif sur les pratiques d'immunisation (ACIP) des États-Unis ait publié ses nouvelles recommandations (12). L'Office régional de la santé du Nord fournit des services à environ 75000 résidents du Nord manitobain, la région la moins peuplée sur les cinq que compte la province, mais qui occupe la plus grande surface géographique. De nombreux habitants vivent dans des collectivités éloignées et isolées, dont certaines ne sont accessibles que par voie aérienne ou maritime. SAVAM a décidé de ne pas recommander l'administration d'une troisième dose du vaccin $R O R$ en raison de la faisabilité opérationnelle d'une telle mesure dans les régions du Nord. Comme le virus circulait dans la région depuis un certain temps, il n'était pas possible de définir une population admissible qui aurait bénéficié d'une troisième dose.

\section{Résultats}

\section{Épidémiologie descriptive}

Cette éclosion d'oreillons s'est déclarée chez des étudiants universitaires de la région de l'Office régional de la santé de Winnipeg. Cette région abrite environ $57 \%$ des 1,4 million de résidents du Manitoba. Trois pics survenus durant les premiers mois de l'éclosion correspondaient à trois évènements importants en matière d'exposition : nombreuses célébrations de retrouvailles à l'Université du Manitoba (du 19 au 25 septembre 2016); fin de semaine de l'Action de grâces (du 8 au 10 octobre), au cours de laquelle les personnes contagieuses ont pu voyager; et Halloween (31 octobre) 
Figure 1a : Cas confirmés d'oreillons $(N=65)$ selon la date d'apparition des symptômes et la région sanitaire, au Manitoba, du 25 septembre au 26 novembre 2016

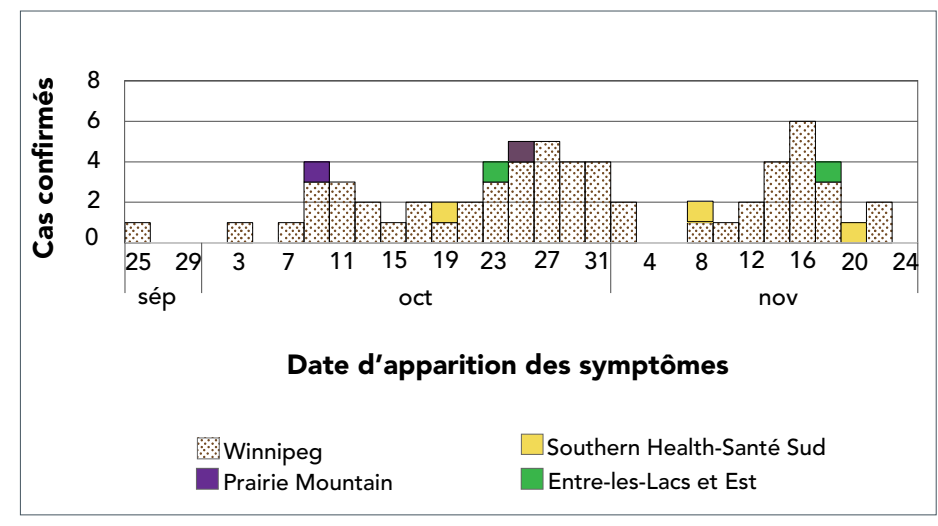

(figure 1a). Durant cette période, les personnes touchées étaient de jeunes adultes de Winnipeg ayant des liens avec des universités, des écoles, des manifestations sportives ou des vacances.

Plus de $80 \%$ des étudiants universitaires nés au Manitoba s'étaient vu administrer deux doses du vaccin contenant le virus des oreillons (données non illustrées).

Fin novembre 2016, l'éclosion s'était propagée à trois régions sanitaires rurales, à savoir la région de l'Office régional de la santé d'Entre-les-Lacs et de l'Est, la région de Southern Health-Santé Sud et la région de Santé de Prairie Mountain, ainsi qu'aux établissements correctionnels situés dans ces régions. En janvier 2017, l'éclosion s'était propagée à la région la plus rurale du Manitoba, celle de l'Office régional de la santé du Nord. Le nombre de cas d'oreillons dans cette région a continué d'augmenter tout au long de l'année, jusqu'à atteindre un pic à la mi-septembre 2017. En comparaison avec les autres régions sanitaires, le taux de morbidité associé aux oreillons dans la région de l'Office régional de la santé du Nord était élevé et soutenu. Malgré l'isolement géographique des collectivités, l'éclosion a continué à se propager dans toute la région, au point de contaminer près de $2 \%$ de sa population.

Après septembre 2017, le nombre de cas d'oreillons au Manitoba a commencé à baisser, en grande partie en raison de la diminution des cas dans la région de l'Office régional de la santé du Nord. SAVAM a annoncé la fin de l'éclosion à la fin 2018, quand il apparut clairement que le niveau d'activité du virus était revenu à la normale (figure $1 \mathrm{~b}$ ).

L'enquête sur l'éclosion a permis de confirmer 2223 cas d'oreillons (1,6 cas pour 1000 personnes). Sur ces 2223 cas d'oreillons, $1566(70,4 \%)$ ont été déclarés dans la région de l'Office régional de la santé du Nord et 370 (16,6\%) dans la région de l'Office régional de la santé de Winnipeg. Les femmes représentaient $48,8 \%(n=1084)$ de tous les cas provinciaux (tableau 1).
Figure 1b : Cas confirmés d'oreillons ( $N=2223$ ) selon le mois d'apparition des symptômes et la région sanitaire, au Manitoba, entre septembre 2016 et décembre 2018

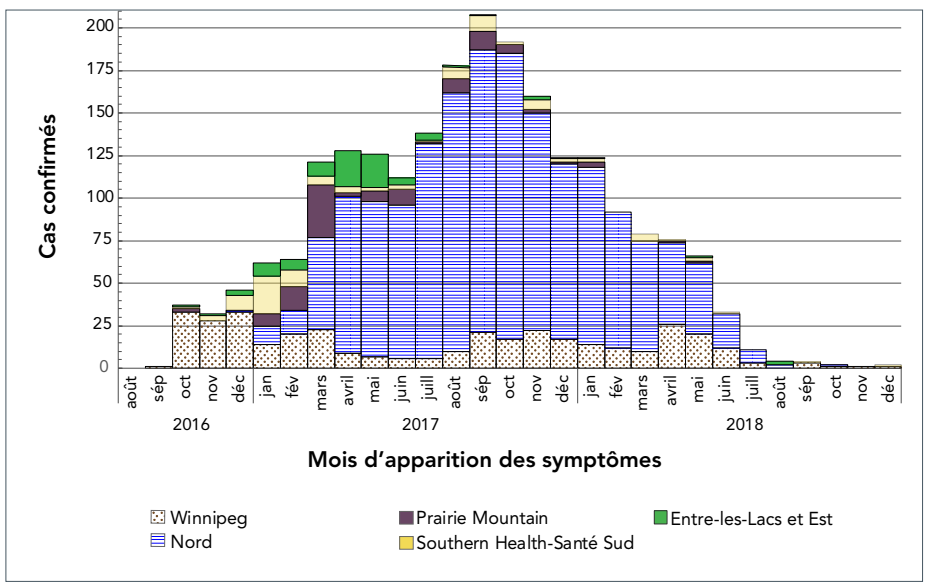

Tableau 1 : Caractéristiques des cas d'oreillons confirmés par l'office régional de la santé, au Manitoba, au Canada, entre septembre 2016 et décembre 2018

\begin{tabular}{|c|c|c|c|c|c|c|}
\hline \multirow[b]{2}{*}{$\begin{array}{l}\text { Caractéris- } \\
\text { tiques }\end{array}$} & \multicolumn{5}{|c|}{ Office régional de la santé } & \multirow[b]{2}{*}{ Total } \\
\hline & Nord & Winnipeg & $\begin{array}{l}\text { Prairie } \\
\text { Mountain }\end{array}$ & $\begin{array}{l}\text { Southern } \\
\text { Health- } \\
\text { Santé Sud }\end{array}$ & $\begin{array}{l}\text { Entre-les- } \\
\text { Lacs et } \\
\text { Est }\end{array}$ & \\
\hline $\begin{array}{l}\text { Incidence } \\
\text { cumulative }^{\text {a }}\end{array}$ & 20,4 & 0,5 & 0,6 & 0,5 & 0,7 & 1,6 \\
\hline Cas (nombre) & 1566 & 370 & 104 & 98 & 85 & 2223 \\
\hline $\begin{array}{l}\text { Pourcentage } \\
\text { du nombre } \\
\text { total de cas }\end{array}$ & 70,4 & 16,6 & 4,7 & 4,4 & 3,8 & 100 \\
\hline $\begin{array}{l}\text { Femme } \\
\text { (nombre) }\end{array}$ & 809 & 164 & 40 & 28 & 43 & 1084 \\
\hline Pourcentage & 51,7 & 44,3 & 38,5 & 28,6 & 50,6 & 48,8 \\
\hline $\begin{array}{l}\text { Âge médian } \\
\text { (années) }\end{array}$ & 26 & 24 & 26 & 24 & 27 & 25 \\
\hline Quartile 1 & 15 & 18 & 19 & 18 & 18 & 17 \\
\hline Quartile 3 & 36 & 33 & 36 & 38 & 40 & 36 \\
\hline $\begin{array}{l}\text { Non vaccinéb } \\
\text { (nombre) }\end{array}$ & 51 & 20 & 3 & 5 & 4 & 83 \\
\hline Pourcentage & 5,9 & 11,4 & 6,4 & 10,9 & 8,3 & 7,0 \\
\hline $\begin{array}{l}\text { Partiellement } \\
\text { vaccinéc } \\
\text { (nombre) }\end{array}$ & 200 & 42 & 10 & 9 & 17 & 278 \\
\hline Pourcentage & 23,1 & 24,0 & 21,3 & 19,6 & 35,4 & 23,5 \\
\hline $\begin{array}{l}\text { Pleinement } \\
\text { vaccinéd }^{\text {(nombre) }}\end{array}$ & 616 & 113 & 34 & 32 & 27 & 822 \\
\hline Pourcentage & 71,0 & 64,6 & 72,3 & 69,6 & 56,3 & 69,5 \\
\hline \multicolumn{7}{|c|}{$\begin{array}{l}\text { a Nombre de cas pour } 1000 \text { personnes } \\
\text { b Aucune dose du vaccin contre la rougeole, les oreillons et la rubéole (ROR) ou du vaccin contre } \\
\text { la rougeole, les oreillons, la rubéole et la varicelle (RORV) n'a été administrée aux patients de } \\
5 \text { à } 29 \text { ans enregistrés auprès de Santé, Aînés et Vie active Manitoba (SAVAM) avant l'âge de } \\
\text { deux mois } \\
\text { c Une dose du vaccin ROR ou du vaccin RORV a été administrée aux patients de } 5 \text { à } 29 \text { ans } \\
\text { enregistrés auprès de SAVAM avant l'âge de deux mois } \\
\text { d Deux doses ou plus du vaccin ROR ou du vaccin RORV ont été administrées aux patients de } \\
5 \text { à } 29 \text { ans enregistrés auprès de SAVAM avant l'âge de deux mois }\end{array}$} \\
\hline
\end{tabular}


Dans l'ensemble, l'incidence était la plus élevée (3,4 cas pour 1000 personnes) chez les personnes de 18 à 29 ans (figure 2). Dans la région de l'Office régional de la santé du Nord, I'incidence chez les personnes de 30 à 45 ans (32,5 cas pour 1000 personnes) était similaire à celle des personnes de 18 à 29 ans (32 cas pour 1000 personnes). L'incidence dans la région de l'Office régional de la santé du Nord (20,4 cas pour 1000 personnes) était largement plus élevée que dans toutes les autres régions $(0,5$ à 0,7 cas pour 1000 personnes).

Figure 2 : Taux d'incidence cumulative de cas d'oreillons pour 1000 personnes, selon le groupe d'âge et l'office régional de la santé, au Manitoba, au Canada, entre septembre 2016 et décembre 2018

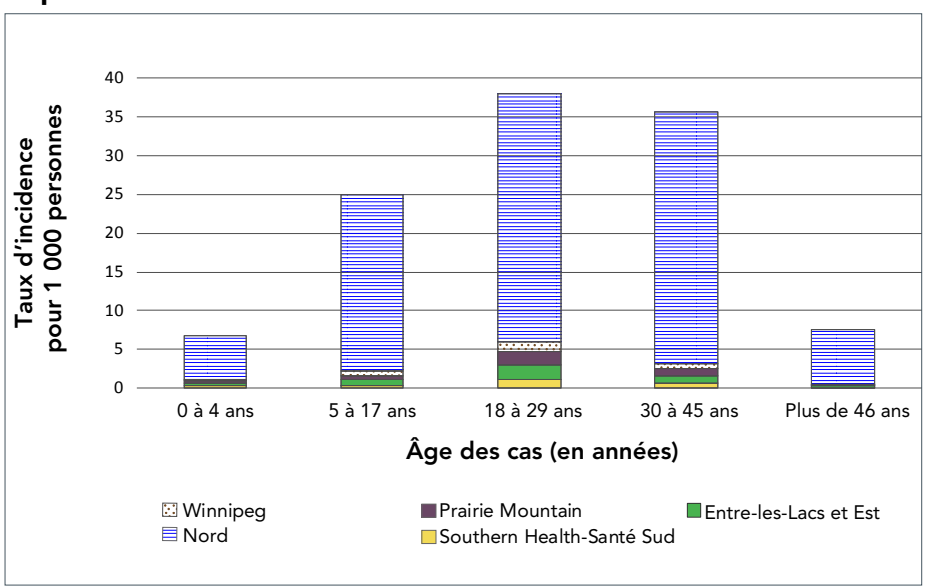

Un cas d'orchite et un cas de méningite, probablement imputables à l'infection aux oreillons, ont été déclarés à SAVAM. Aucun décès n'a été signalé.

\section{Résultats de laboratoire}

La majorité des cas confirmés $(97,1 \%)$ ont été confirmés en laboratoire, dont 87,8 \% par la technique de RT-PCR, 9,2 \% par sérologie et $0,1 \%$ par culture virale. De plus, $2,8 \%$ des cas de patients symptomatiques ont été confirmés par l'existence d'un lien épidémiologique avec des cas confirmés en laboratoire.

Les échantillons ont d'abord été transmis au LNM en vue de leur génotypage. Après que le nombre d'échantillons ait dépassé les capacités du LNM, environ $10 \%$ d'échantillons choisis au hasard ont été cultivés et génotypés. Sur ces 243 échantillons, $229(94,2 \%)$ se sont révélés appartenir au génotype G, le génotype endémique du virus des oreillons en circulation au Canada et aux États-Unis (12). Les échantillons restants n'ont pas pu être séquencés.

\section{Situation vaccinale}

Les antécédents de vaccination des personnes de moins de 30 ans qui vivent au Manitoba depuis leur naissance étaient accessibles dans le registre provincial d'immunisation de la population. D'après le registre, sur les 1183 (53,2 \%) personnes de 5 à 29 ans admissibles à deux doses du vaccin contenant le virus des oreillons au Manitoba et enregistrées auprès de SAVAM avant l'âge de deux mois, 822 (69,5\%) avaient reçu au moins deux doses (tableau 1). Le taux de couverture des cas d'oreillons avec l'administration de deux doses du vaccin contre les oreillons allait de 56,3 \% dans la région d'Entre-les-Lacs et de l'Est à $72,3 \%$ dans la région de Prairie Mountain. Sur les 822 personnes qui ayant reçu au moins deux doses du vaccin contenant le virus des oreillons, l'intervalle médian entre l'administration de la dernière dose et l'apparition des symptômes était de 11,3 années.

\section{Discussion}

SAVAM a dirigé une enquête lors d'une éclosion d'oreillons qui a touché la province entre septembre 2016 et décembre 2018. Selon la Division de la surveillance et de l'épidémiologie de l'Agence de la santé publique du Canada, en janvier 2019, cette éclosion était, au vu du nombre de cas signalés, la plus importante que le Canada ait connue au cours des vingt dernières années. Cette éclosion s'est déclarée chez des étudiants universitaires qui présentaient une couverture vaccinale élevée, avec l'administration de deux doses du vaccin contre les oreillons, puis s'est étendue à d'autres collectivités du Manitoba, la propagation ayant sans doute été facilitée par des événements sociaux. Le niveau d'activité était particulièrement élevé et soutenu dans la région de l'Office régional de la santé du Nord, la région la plus rurale du Manitoba où se trouvent de nombreuses collectivités isolées.

Cette éclosion a mis au jour une vaste population sensible malgré la disponibilité, depuis trente ans, d'un programme de vaccination contre les oreillons financé par les fonds publics. Le faible taux de couverture vaccinale contre les oreillons a probablement contribué à cette éclosion, bien que l'ampleur de cette contribution ait varié d'une région à l'autre. La couverture vaccinale contre les oreillons au Manitoba est restée inférieure au taux de $92 \%$ nécessaire pour assurer et préserver l'immunité collective (13). Selon le registre provincial d'immunisation, en 2017, 90 \% des personnes de 17 ans ayant vécu au Manitoba sans interruption depuis leur naissance s'étaient vu administrer deux doses du vaccin contenant le virus des oreillons, contre $31 \%$ des personnes de 17 ans n'ayant pas vécu au Manitoba de manière continue depuis leur naissance, une situation qui s'explique en partie par le caractère incomplet des données du registre sur les résidents qui ne sont pas nés au Manitoba (14).

Les personnes touchées étaient notamment de jeunes adultes vaccinés, ce qui laisse croire que la baisse de l'immunité conférée par la vaccination a sans doute contribué davantage à cette éclosion. Près de trois-quarts (70 \%) des personnes dont les données de vaccination étaient consignées dans le registre provincial étaient totalement immunisées. Cependant, dans le cas des personnes qui se sont vu administrer, preuves à l'appui, au moins deux doses du vaccin contenant le virus des oreillons, 
une durée médiane de plus de onze années s'était écoulée depuis l'administration de la dernière dose. Cela concorde avec les autres rapports faisant état d'une baisse de l'immunité conférée par la vaccination contre les oreillons (15-17).

Cette baisse de l'immunité pourrait être imputable à l'absence de stimulation naturelle résultant d'une exposition au virus sauvage des oreillons $(18,19)$. Contrairement aux autres provinces et territoires canadiens qui signalent des éclosions d'oreillons de moindre envergure depuis les années 1980, les signalements de cas d'oreillons ont toujours été rares au Manitoba (7). Dans le Nord manitobain, où la densité de population est faible et la couverture vaccinale élevée, la stimulation naturelle résultant d'une exposition au virus a encore moins de chance de survenir que dans les autres régions du Manitoba.

De plus, la souche du virus des oreillons dans le vaccin nordaméricain (Jeryl-Lynn) est de génotype A. Le remplacement du virus des oreillons de génotype $A$, à une époque où le vaccin n'existait pas, par le virus des oreillons de génotype $G$, actuellement endémique au Canada et aux États-Unis, pourrait également contribuer à la baisse de l'immunité (20).

Le nombre de complications signalées en lien avec cette éclosion d'oreillons était faible, ce qui pourrait traduire des lacunes dans la surveillance de la santé publique, car l'étude des cas pourrait avoir été effectuée avant que les complications se manifestent. Il est possible que la vaccination précédente contre les oreillons ait conféré une certaine protection contre les maladies graves $(21,22)$. Étant donné que les complications étaient rarement signalées, il n'a pas été possible d'analyser l'efficacité du vaccin contre les complications graves liées aux oreillons.

\section{Limitations}

Les antécédents de vaccination de certaines personnes n'étaient pas disponibles dans le registre provincial d'immunisation. II se peut donc que le taux de couverture vaccinale des cas d'oreillons ait été supérieur. Le cas échéant, un faible taux de couverture vaccinale pourrait moins avoir contribué à cette éclosion que la baisse de l'immunité conférée par la vaccination. Dans une prochaine étude, l'équipe de recherche envisage d'examiner la relation entre le moment où la dernière dose du vaccin contenant le virus des oreillons a été administrée et celui où la maladie des oreillons s'est manifestée.

\section{Conclusion}

Un effort de santé publique important et soutenu a été nécessaire tout au long de cette éclosion, qui s'est déclarée chez des étudiants universitaires puis s'est propagée à toute la province. Cela souligne la nécessité d'atteindre et de maintenir un taux de couverture élevé dans la population, avec l'administration de deux doses du vaccin contenant le virus des oreillons. En raison de la baisse de l'immunité conférée par la vaccination, il se peut que la population compte encore un vaste groupe de personnes sensibles. La possibilité d'administrer une troisième dose du vaccin contenant le virus des oreillons pourrait être envisagée lors de futures éclosions pour stimuler l'immunité conférée par la vaccination dans le cas où les données épidémiologiques le justifieraient.

\section{Déclaration des auteurs}

Y. W. - Conceptualisation, méthodologie, enquête, analyse formelle, rédaction - rédaction de la version préliminaire révision et édition

K. W. - Conceptualisation, méthodologie, rédaction - révision et édition

R. R. - Méthodologie, rédaction - révision et édition

K. K. - Méthodologie, rédaction - révision et édition

C. L. - Supervision, rédaction - révision et édition

\section{Conflit d'intérêts}

Les auteurs n'ont déclaré aucun conflit d'intérêts potentiel.

\section{Remerciements}

Nous tenons à remercier les personnes suivantes pour leur contribution : N. Casaclang, D. Race, I. Hossack, T. Hilderman de Santé, Aînés et Vie active Manitoba; K. Dust du Laboratoire provincial de Cadham (Manitoba); D. Mac-Donald et M. Roy de l'Agence de la santé publique du Canada.

\section{Financement}

Aucun financement externe n'a été obtenu pour la réalisation de ce rapport.

\section{Références}

1. Heymann DL, editor. Control of Communicable Diseases Manual, 20th ed. Washington (DC): American Association of Public Health; 2014.

2. Hamborsky J, Kroger A, Wolfe S, editors. Epidemiology and prevention of vaccine-preventable diseases, 13 ed. Washington (DC): Centers for Disease Control and Prevention; 2015.

3. Agence de la santé publique du Canada. Lignes directrices pour la prévention et le con-trôle des éclosions d'oreillons au Canada. Relevé des maladies transmissibles au Canada 2010 Jan;36 Suppl 1:1-51. DOI

4. Government of Manitoba. The Public Health Act (C.C.S.M. c. P210): Reporting of Diseases and Conditions Regulation, 2019. https://web2.gov.mb.ca/laws/statutes/ccsm/p210e. php

5. L'histoire de la santé publique : chronologie de I'immunisation. Ottawa (ON): Association canadienne de santé publique; (Accédé 2019-10-18). https://www.cpha.ca/ $\mathrm{fr} /$ chronologie-de-limmunisation 
6. Comité consultatif national de l'immunisation (CCNI). Déclaration sur le vaccin contre les oreillons. Relevé des maladies transmissibles au Canada 2007;33(ACS-8):110. https://www.canada.ca/en/public-health/services/ reports-publications/canada-communicable-disease-report$\mathrm{ccdr} /$ monthly-issue/2007-33/statement-on-mumps-vaccine. html

7. Manitoba annual summary of communicable diseases 2015. Winnipeg (MB): Government of Manitoba; 2017.

8. Maladies évitables par la vaccination : Rapport de surveillance en date du 31 décembre 2015. Ottawa (ON) : Agence de la santé publique du Canada; 2017. https://www. canada.ca/fr/sante-publique/services/publications/vie-saine/ maladies-evitables-vaccination-rapport-surveillance-31decembre-2015.html

9. Health, Seniors and Active Living: Regional health authorities in Manitoba. Winnipeg (MB): Government of Manitoba; (Accédé 2019-03-01). https://www.gov.mb.ca/health/rha/ index.html

10. Mumps outbreak and testing processes. Winnipeg (MB): Communicable Disease Control, Government of Manitoba. 2017. https://www.gov.mb.ca/health/publichealth/cdc/ protocol/mumps.pdf

11. Manitoba's immunization program: vaccines offered free-ofcharge (eligibility criteria for publicly-funded vaccines). Winnipeg (MB): Government of Manitoba; 2019 (Accédé 2019-03-01). https://www.gov.mb.ca/health/publichealth/ cdc/vaccineeligibility.html

12. Marin $M$, Marlow $M$, Moore KL, Patel M. Recommendation of the Advisory Committee on Immunization Practices for use of a third dose of mumps virus-containing vaccine in persons at increased risk for mumps during an outbreak. MMWR Morb Mortal Wkly Rep 2018 Jan;67(1):33-8. DOI PubMed

13. Quinlisk MP. Mumps control today. J Infect Dis 2010 Sep;202(5):655-6. DOI PubMed

14. Annual report of immunization surveillance, 2017. Winnipeg (MB): Government of Manitoba; 2019 (Accédé 2019-12-19). https://www.gov.mb.ca/health/publichealth/surveillance/ immunization/index.html
15. Marin M, Quinlisk P, Shimabukuro T, Sawhney C, Brown C, Lebaron $\mathrm{CW}$. Mumps vaccination coverage and vaccine effectiveness in a large outbreak among college students-lowa, 2006. Vaccine $2008 \mathrm{Jul} ; 26(29-30): 3601-7$.

DOI PubMed

16. Davidkin I, Jokinen S, Broman M, Leinikki P, Peltola H. Persistence of measles, mumps, and rubella antibodies in an MMR-vaccinated cohort: a 20-year follow-up. J Infect Dis 2008 Apr;197(7):950-6. DOI PubMed

17. de Wit J, Emmelot ME, Poelen MC, van Binnendijk RS, van der Lee S, van Baarle D, Han WG, van Els CA, Kaaijk $P$. Mumps infection but not childhood vaccination induces persistent polyfunctional CD8+ T-cell memory. J Allergy Clin Immunol 2018 May;141(5):1908-1911.e12. DOI PubMed

18. Dayan GH, Quinlisk MP, Parker AA, Barskey AE, Harris ML, Schwartz JM, Hunt K, Finley CG, Leschinsky DP, O'Keefe AL, Clayton J, Kightlinger LK, Dietle EG, Berg J, Kenyon CL, Goldstein ST, Stokley SK, Redd SB, Rota PA, Rota J, Bi D, Roush SW, Bridges CB, Santibanez TA, Parashar U, Bellini WJ, Seward JF. Recent resurgence of mumps in the United States. N Engl J Med 2008 Apr;358(15):1580-9. DOI PubMed

19. Barskey AE, Glasser JW, LeBaron CW. Mumps resurgences in the United States: A historical perspective on unexpected elements. Vaccine 2009 Oct;27(44):6186-95. DOI PubMed

20. Dayan GH, Rubin S, Plotkin S. Mumps outbreaks in vaccinated populations: are available mumps vaccines effective enough to prevent outbreaks? Clin Infect Dis 2008 Dec;47(11):1458-67. DOl PubMed

21. Sane J, Gouma S, Koopmans M, de Melker H, Swaan C, van Binnendijk R, Hahné S. Epidemic of mumps among vaccinated persons, The Netherlands, 2009-2012. Emerg Infect Dis 2014 Apr;20(4):643-8. DOI PubMed

22. Zamir CS, Schroeder H, Shoob H, Abramson N, Zentner G. Characteristics of a large mumps outbreak: clinical severity, complications and association with vaccination status of mumps outbreak cases. Hum Vaccin Immunother 2015;11(6):1413-7. DOI PubMed 